\title{
Performance Analysis of Faulty Gallager-B Decoding of QC-LDPC Codes with Applications
}

\author{
Omran Al Rasheed, Srdjan S. Brkic, Student Member, IEEE, \\ Predrag N. Ivanis, Member, IEEE, and Bane Vasic, Fellow IEEE
}

\begin{abstract}
In this paper we evaluate the performance of Gallager-B algorithm, used for decoding low-density paritycheck (LDPC) codes, under unreliable message computation. Our analysis is restricted to LDPC codes constructed from circular matrices (QC-LDPC codes). Using Monte Carlo simulation we investigate the effects of different code parameters on coding system performance, under a binary symmetric communication channel and independent transient faults model. One possible application of the presented analysis in designing memory architecture with unreliable components is considered.
\end{abstract}

Keywords - faulty hardware, Gallager-B decoder, Monte Carlo simulation, QC-LDPC codes.

\section{INTRODUCTION}

$\mathrm{L}^{\mathrm{s}}$ OW-density parity-check (LDPC) codes are powerful error correction codes that achieve a performance near the Shannon limit [1]. They have received significant practical interest and have been adopted in many telecommunications standards. The LDPC codes can be efficiently decoded by message passing iterative decoders, whose realization complexity increases linearly with code length [2].

According to a new design paradigm for VLSI (Very Large Scale Integration) technologies, fully reliable operations are not guaranteed [3]. New nano-scale technologies are more sensitive to noise, which appears as a consequence of radiation or electromagnetic interference. Thus, the analysis of different decoding algorithms under unreliable hardware is meaningful. A hardware component is assumed to be unreliable if it is

Paper received April 4, 2014; revised Jun 11, 2014; accepted July 4, 2014. Date of publication July 31, 2014. The associate editor coordinating the review of this manuscript and approving it for publication was Prof. Zorica Nikolić.

This paper is a revised and expanded version of the paper presented at the 21th Telecommunications Forum TELFOR 2013.

This work was supported by the Seventh Framework Programme of the European Union, under Grant Agreement number 309129 (i-RISC project). It is also funded in part by the NSF under grant CCF-0963726.

Omran Al Rasheed is with the School of Electrical Engineering, University of Belgrade, Bul. kralja Aleksandra 73, 11120 Belgrade, Serbia (e-mail: omrano84@hotmail.com).

Srdjan S. Brkic is with the Innovation Centre of the School of Electrical Engineering, University of Belgrade, Bul. kralja Aleksandra 73, 11120 Belgrade, Serbia (e-mail: brka05@gmail.com).

Predrag N. Ivaniš is with the School of Electrical Engineering, University of Belgrade, Bul. kralja Aleksandra 73, 11120 Belgrade, Serbia (phone: +381113218381; e-mail: predrag.ivanis@etf.rs).

Bane Vasic is with the University of Arizona, AZ 85721 USA, (email: vasic@ece.arizona.edu). subject to so-called transient faults, i.e. faults that manifest themselves at particular time instants but do not necessarily persist for later times [4]. These faults have probabilistic behavior and can be described statistically through erroneous component output probability.

Recently, different noisy LDPC decoders have been analyzed by using simulation, density evolution or EXIT chart tools. The performance of LDPC codes under faulty Gallager-A and belief propagation decoding were determined in [5], using a density evolution method. A similar analysis using EXIT function is provided in [6], for Gallager-B algorithm. Also, a probabilistic analysis of Gallager-B decoding algorithm was presented in [7]. More general finite-alphabet decoders were investigated in [8], while noisy min-sum decoder realization was considered in [9].

In this paper, we present our first results in the empirical evaluation of the performance of LDPC codes constructed from circular matrices (quasi-cyclic LDPC codes) decoded using Gallager-B decoder, built from unreliable components. We examine the influence of different code parameters, decoder structures and fault model parameters on overall system performance in order to gain an insight into the relative importance of failures in different logic gates and their relation with parameters such as code length and number of iterations.

Finally, it will be shown that the obtained numerical results can be applied for designing memory architecture with unreliable components. In particular, it will be shown that the decision probabilities during the decoding process are incorrect can be related with the probabilities of failures in the particular gates of decoder combinatorial logic.

The rest of the paper is organized as follows. In Section II, the construction method for QC-LDPC codes is described. In Section III we give a description of faulty Gallager-B decoder. Section IV presents the numerical results. Finally, some concluding remarks and future research directions are given in Section V.

\section{Construction OF QC-LDPC CODES}

In general, the LDPC codes can be constructed by pseudorandom or algebraic methods [10]-[11]. Algebraic constructions of LDPC codes can be performed based on finite geometries, what is described in [12] and [13], or circulant permutation matrices [14]. By using the second approach, so-called quasi-cyclic (QC) LDPC codes are constructed. In this section, we explain the construction principle of parity check matrix of QC-LDPC codes. 
The principal property of QC-LDPC codes is that their parity check matrix consists of circulant submatrices, which could be either based on the identity matrix [15] or a smaller random matrix [16]. The main advantage of this construction principle compared to randomly constructed codes is that QC-LDPC encoding procedure is easier to implement [17]. The encoder of QC-LDPC codes can be implemented by using a series of shift registers, which allows its complexity to be proportional to code length [18]. We next present one method for the construction of regular QC-LDPC codes, originally presented in [14].

The parity check matrix $\mathbf{H}$ of a QC-LDPC code is constructed by a concatenation of circulant submatrices, as shown in the following

$$
\mathbf{H}=\left[\begin{array}{ccccc}
I_{1} & I_{a} & I_{a^{2}} & \cdots & I_{a^{k-1}} \\
I_{b} & I_{a b} & I_{a^{2} b} & \cdots & I_{a^{k-1} b} \\
\cdots & \cdots & \cdots & \cdots & \cdots \\
I_{b^{j-1}} & I_{a b^{j-1}} & I_{a^{2} b^{j-1}} & \cdots & I_{a^{k-1} b^{j-1}}
\end{array}\right],
$$

where $I_{x}$ represents an identity matrix whose rows are cyclically shifted to the left by positions $x$, and parameters $a$ and $b$ are two nonzero elements with multiplicative orders $o(a)=d_{c}$ and $o(b)=d_{v}$, respectively, where $d_{c}$ and $d_{v}$ denote the weight of each row and column of matrix $\mathbf{H}$, respectively. The parameters $a$ and $b$ should be chosen from the Galois field $\operatorname{GF}(m)$, where $m$ is a prime number. In the simulation analysis presented in this paper we consider two QC-LDPC codes. The first code is constructed by choosing $m=31, a=2, b=5$, which produces a regular code with parameters $d_{c}=5$ and $d_{v}=3$, and parity check matrix dimensions $93 \times 155$ (code length is equal to $n=155$ bits). It is the so-called Tanner code $(155,64)$. The second code is based on $m=61, a=2, b=5$ and has a row weight $d_{c}=5$, column weight $d_{v}=3$, and parity check matrix dimensions $183 \times 305$ (code length is equal to $n=305$ bits).

\section{Description OF FAulty GALlaGer-B DECODER}

\section{A) Noise free Gallager-B decoder}

Decoding procedures of LDPC codes are usually described by the Tanner graph representation. The Tanner graph is a bipartite graph constructed from two sets of nodes - variable (bit) nodes and check nodes. Nodes, from a different set, connected to a single node, are referred to as its neighbors. The degree of a node is the number of its neighbors. In a $\left(d_{v}, d_{c}\right)$ regular LDPC code, each variable node has a degree $d_{v}$ and every check node degree is $d_{c}$.

The Gallager-B algorithm represents an iterative decoding procedure operating in a binary field. During every decoding iteration, binary messages are sent along the edges of Tanner graph. Let $E(x)$ represent a set of edges incident on a node $x$ ( $x$ can be either variable or check node). Let $m_{i}(e)$ and $m_{i}^{\prime}(e)$ denote the messages sent on edge $e$ from a variable node to a check node and a check node to a variable node at iteration $i$, respectively. If we denote the initial value of a bit at a variable node $v$ as $r(v)$, the Gallager-B algorithm can be summarized as follows [19].

Initialization $(i=1)$ : For each variable node $v$, and each set $E(v)$, messages sent to check nodes are computed as follows

$$
m_{1}(e)=r(v) .
$$

Step (i) (check-node update): For each parity check node $c$ and each set $E(c)$, update rule for $i$-th iteration, $i>1$, is defined as follows

$$
m_{i}^{\prime}(e)=\left(\sum_{e^{\prime} \in E(c) \backslash\{e\}} m_{i-1}(e)\right) \bmod 2 .
$$

Step (ii) (variable-node update): For each variable node $v$ and each set $E(v)$, update rule for $i$-th iteration, $i>1$, is defined as follows

$$
m_{i}(e)=\left\{\begin{array}{ccc}
1, & \text { if } & \sum_{e^{\prime} \in E(v) \backslash\{e\}} m_{i}^{\prime}(e) \geq b_{i} \\
0, \text { if } & d_{v}-1-\sum_{e^{\prime} \in E(v) \backslash\{e\}} m_{i}^{\prime}(e) \leq b_{i}, \\
r(v), & \text { otherwise }
\end{array}\right.
$$

where $b_{i}$ represents a threshold dependent on iteration $i$. In our analysis we considered a constant threshold value $b_{i}=\left\lceil\frac{d_{v}}{2}\right\rceil, i>1$.

Step (iv) (decision): After a predefined number of iterations the final decision of transmitted bit $\widehat{v}$ is made on the basis of majority of its estimates $m_{i}(e), e \forall E(v)$.

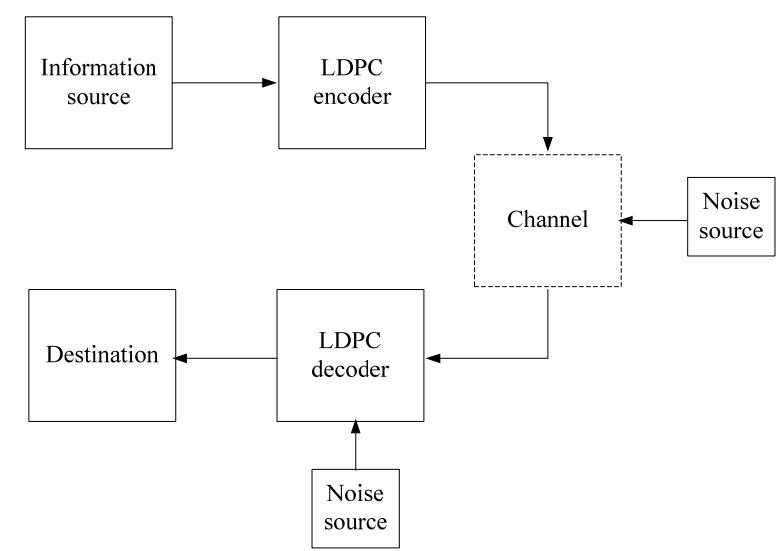

Fig. 1. Schematic diagram of an information system that processes unreliable signals with unreliable circuits.

\section{B) Faulty Gallager-B decoder}

We study the performance of a faulty Gallager-B decoder in the presence of transient faults. As illustrated in Fig 1, originally presented in [5], besides noise that exists in a communication channel, errors are inserted by the LDPC decoder itself. We assume an independent transient faults model in which errors occur at the Tanner graph level of implementation. In other words, every edge in the Tanner graph behaves as a binary symmetric channel (BSC) with some crossover probability. The probability that a message originating from a variable node is incorrect is denoted as $p$, while crossover probability in BSC that corresponds to check node message transition is equal to $q$, as can be seen in Fig. 2. Assigning different crossover probabilities enables us to determine the influence of faults in different nodes on overall decoder performance. 


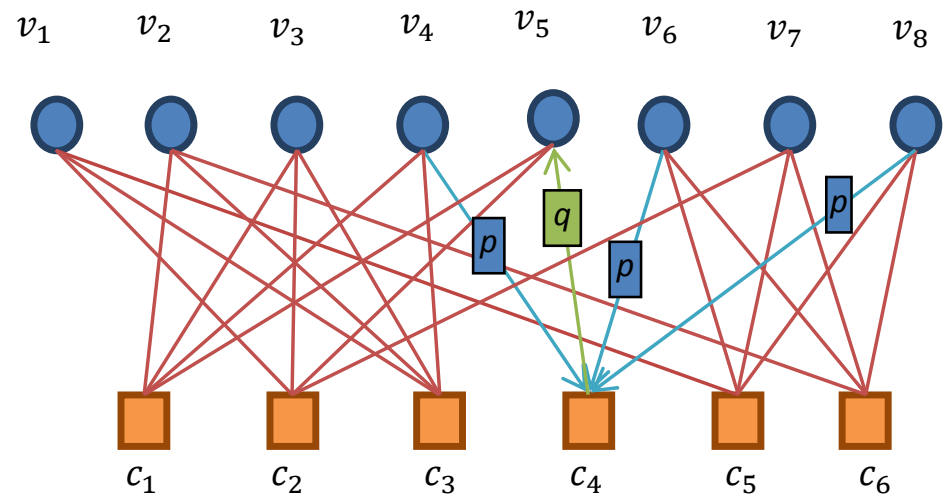

Fig. 2. Bipartite graph with faulty decoder.

\section{NuMERICAL RESUlts}

In this section we present the performance analysis of faulty Gallager-B decoder, described in the previous section. The two QC-LDPC codes have been examined and their performances are compared for several implementations of faulty Gallager-B decoders. All numerical results presented in this section have been obtained by Monte Carlo simulations.

The sequence of all-zero codewords is transmitted through BSC with a predefined crossover probability and then decoded by a faulty iterative decoder. As described earlier, messages that are passed between nodes can be faulty. The message $m_{i}(e)$ passes through the noise channel with error probability $p$, thus, a bit estimate can be erroneous as a consequence of a majority of unsatisfied parity checks or the faults in variable node implementation or both. Similarly, due to BSC crossover probability $q$, message $m_{i}^{\prime}(e)$ may incorrectly inform a variable node is the parity check equation satisfied or not.

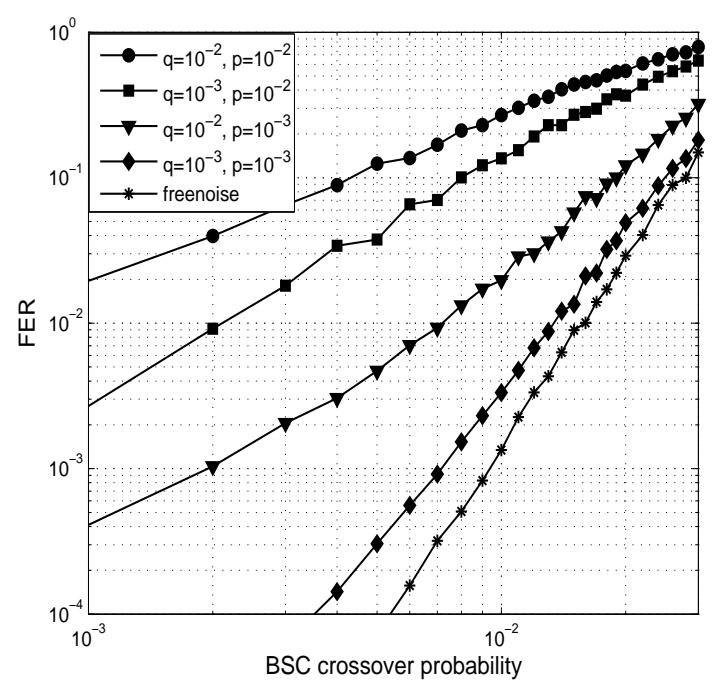

Fig. 3. Performance of Tanner code $(155,64)$ decoded by a faulty Gallager-B decoder, five iterations.

First, we evaluate the performance of Tanner code (with $n=155, d_{v}=3$ and $d_{c}=5$ ) decoded by a faulty Gallager-B decoder. The code frame error rate (FER) performance is given as a function of communication channel crossover probability. FER curves for several values of decoder failures probabilities $p$ and $q$, when 5 decoding iterations are performed, are presented in Fig. 3. It can be observed that decoder failures greatly degrade the frame error rate, but failures in variable and check nodes have different influence on the code performance. The simulation has shown that the decoder is more sensitive to errors that occur in variable nodes. The main reason for this behavior is related to variable node ability to compensate the parity check failures. The majority voting conducted in variable nodes can correct a fraction of parity check failures. However, if a variable node output is erroneous the correction ability of a decoder is decreased.

Finally, the presented results indicate that the decoder performance can be significantly improved by better protection of variable nodes (e.g. by making the majority voting gates that perform the operation in (4) more reliable).

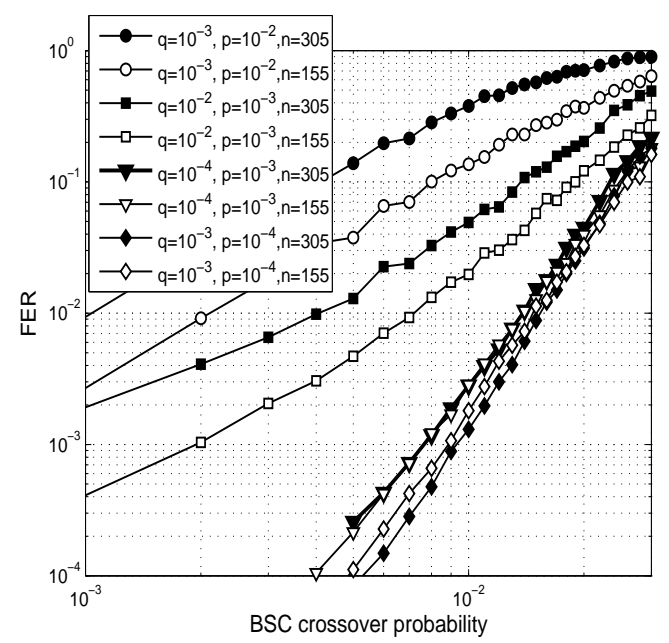

Fig. 4. Performance comparison of two QC-LDPC codes with different lengths ( $n=155$ and $n=305)$ with $d_{c}=5, d_{v}=3$, decoded by a faulty Gallager-B decoder in five iterations.

We also evaluate the performance of two QC-LDPC codes with code lengths $n_{1}=155$ and $n_{2}=305$, with the same parameters $d_{v}=3$ and $d_{c}=5$. Performance comparison is illustrated in Fig. 4. Although the code with longer codewords has better correcting capabilities, it is also more prone to processing errors. The simulation has shown, that when the errors inserted into decoder are 
frequent ( $p=10^{-2}$ or $q=10^{-2}$ ), longer code length may have a negative impact on overall performance. Thus, a code with length $n_{1}=155$ achieves a lower FER, compared to a code with length $n_{2}=305$ even for the case $p=10 q=10^{-3}$. However, a longer code achieves a lower FER when hardware faults are rare and variable nodes are more reliable $\left(q=10 p=10^{-3}\right)$.

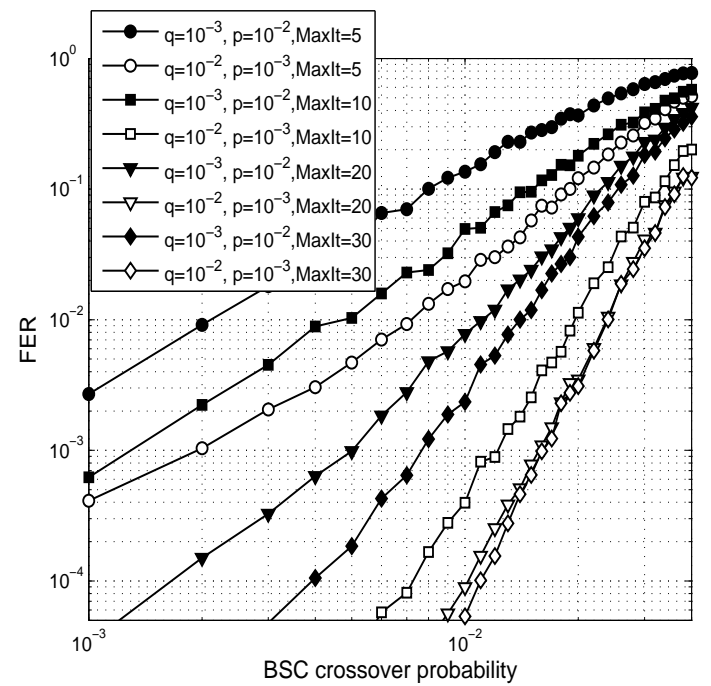

Fig. 5. Influence of the number of decoding iterations on faulty Gallager-B decoder performance, Tanner code $(155,64)$.

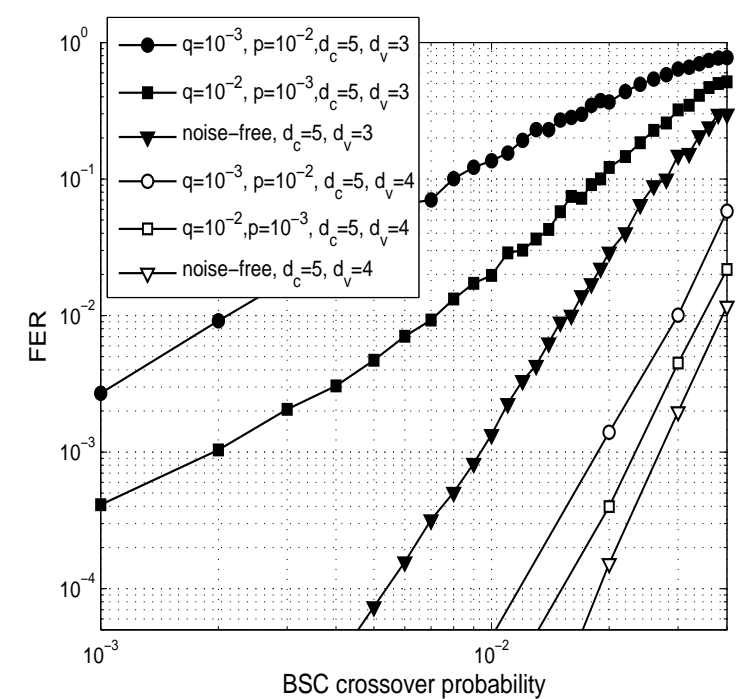

Fig. 6. Performance comparison of two QC-LDPC codes of codeword length $n=155$ with $d_{c}=5$ and different column weight ( $d_{v}=3$ and $\left.d_{v}=4\right)$, faulty decoding in five iterations.

The performance of the Gallager-B algorithm depends on the number of iterations [10], thus assessing the effect of the number of iterations of faulty decoder is meaningful. The performances of a faulty decoder, when different numbers of decoding iterations are used, are presented in Fig. 5. It is obvious that increasing the number of decoding iterations leads to lower error rates. However, it can be noticed that the improvement depends on the structure of the errors that exist in decoder.

If the faults in variable nodes are dominant $\left(p=10^{-2}\right.$, $q=10^{-3}$ ), performances can be improved significantly by increasing the number of iterations. In contrast, the FER levels are much lower if the check nodes faults have a dominant effect and cannot be improved significantly by increasing the number of iterations. For example, when the Tanner code is decoded by a faulty Gallager-B decoder with parameters $p=10^{-3}$ and $q=10^{-2}$ only 10 decoding cycles are sufficient and the error rate does not improve further.

Finally, we investigate the influence of code rate on decoder performance. We compare the error rates of two QC-LDPC codes with the same length $(n=155)$ and check node degree $\left(d_{c}=5\right)$, but different variable node degrees $\left(d_{v}=3\right.$ or $\left.d_{v}=4\right)$. The obtained results are presented in Fig. 6 . The code with higher variable nodes degree (lower code rate) can correct more errors that appear in a communication channel, but the decoder is also more complex and more prone to errors. It is interesting to notice that the performance of a code with a lower code rate is less degraded by decoder failures.

\section{ApPlicAtions in Design of MEMORY ARCHITECTURES WITH UNRELIABLE COMPONENTS}

In this section we will describe a memory architecture based on the Taylor-Kuznetzov (TK) scheme [20-22]. As the equivalence between TK scheme and Gallager-B decoding algorithm is well known and described in [2325], the above analysis can be directly applied for the performance analysis of this memory architecture.

After encoding, $d_{c}$ copies of every coded bit $v_{i}$ are stored in separate registers. Although bit-copies initially have the same values, after some time they can be different, as the registers are made from memory cells that are considered to be unreliable.

New estimates of each of these copies are obtained by using one combination of $d_{c}-1$ checks. The estimates are obtained by using an iterative procedure that consists of the next steps:

1. Evaluate an estimate for each bit-copy (exclude one distinct parity check from the original set of check for each bit-copy). By using the variable bits $v_{k}^{(n)}$ (the $k$-th variable bit in the $n$-th register), the $n$-th parity check bit is calculated. Every register is used to calculate one parity check bit only. For instance, the first parity check is calculated by using the first register, the corresponding value $m_{2}^{\prime}\left(c_{1}->v_{5}\right)$ is equal to zero if this parity check is satisfied.

2. The value of bit-copy is defined by a majority of its estimates (the decision element in this case is a majority logic gate). Therefore, a new value of the $k$-th variable bit in the $n$-th register is estimated by using parity checks obtained from the other registers. The output of this step is the value of the variable bit, that will be used in the next iteration.

The above procedure is illustrated in Fig. 7, for the case of codeword length $n=8$ and code structure described by using the Tanner graph from Fig 2. It can be noticed that the 5-th bit in the first register is updated without using the estimation $m_{2}^{\prime}\left(c_{1}->v_{5}\right)$, obtained from the first register by using values from the previous iteration. 
If the memory is unreliable, all registers are faulty with the probability $P_{R}$, XOR gates are faulty with probability $P_{X}$ and ML gates' probability of failure is $P_{M}$. It can be noticed that variable nodes are incorrect if ML circuit output is faulty or an error occurs in registers, i.e.

$$
p=\left(1-P_{R}\right) P_{M}+\left(1-P_{M}\right) P_{R} \text {. }
$$

On the other hand, the parity check nodes can be incorrect only if the XOR gates are faulty, i.e.

$$
q=P_{X} \text {. }
$$

By using the above identities, we can establish relations between the probabilities in Gallager-B algorithm and physical properties of the logic gates in TK memory architecture.
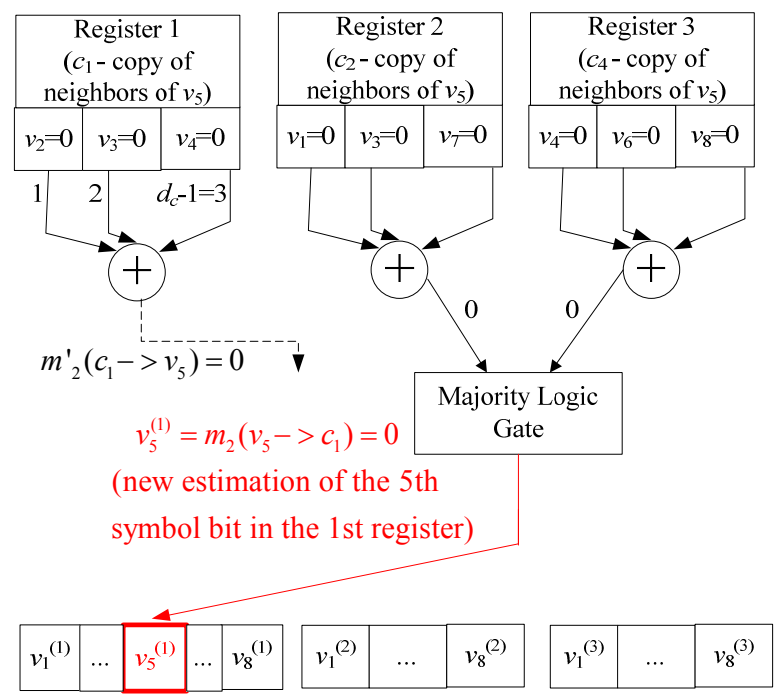

Fig. 7. Taylor-Kuznetsov memory architecture that corresponds to the bipartite graph from Fig. 2.

\section{CONCLUSION}

In this paper, we have evaluated the performance of QC-LDPC codes decoded by a faulty Gallager B decoder. The influence of code length, code rate and the number of decoding iterations on coding system performance is analyzed. Particularly important is the analysis of the influence of failures in different parts of a decoder. It enables us to determine the most sensitive structures in a decoder and make them more reliable.

Furthermore, the application of the described algorithm in the design of memory architecture with unreliable components is considered. Using the well-known equivalence between faulty Gallager-B algorithm and TK scheme, we have found the connection between the physical properties of logic gates (XOR, ML) and probabilities that variable and parity check nodes are faulty. According to the obtained numerical results, it can be concluded that it is important to use reliable $\mathrm{ML}$ circuits and registers, while XOR gates with lower reliability will not significantly affect the system performances.

\section{REFERENCES}

[1] H. D. Pfister, I. Sason, and R. Urbanke, "Capacity achieving ensembles for the binary erasure channel with bounded complexity," IEEE Transactions on Information Theory, vol. 51, no. 7, pp. 2352-2379, July 2005.

[2] R. G. Gallager, "Low Density Parity Check Codes", Cambridge, MA: M.I.T Press, 1963.
[3] S. Ghosh, K. Roy, "Parameter variation tolerance and error resiliency: New design paradigm for the nanoscale era," Proceedings of IEEE, vol. 98, no. 10, pp. 1718-1751, Oct. 2010.

[4] C. N. Hadjicostis and G. C. Verghese, "Coding Approaches to Fault Tolerance in Linear Dynamic Systems," IEEE Transactions on Information Theory, vol. 51, no. 1, pp. 210-228, Jan. 2005.

[5] L. Varshney, "Performance of LDPC codes under faulty iterative decoding," IEEE Transactions on Information Theory, vol. 57, no. 7, pp. 4427-4444, July 2011.

[6] F. Leduc-Primeau and W. Gross, "Faulty Gallager-B decoding with optimal message repetition," in Proc 50th Allerton Conf. Communication, Control, and Computing, 2012, pp. 549-556.

[7] S. M. SadeghTabatabaei Yazdi, H. Cho, L. Dolecek, "Gallger B decoder on noisy hardware," IEEE Transactions on Communications, vol. 61, no. 5, pp. 1660-1673, May 2013.

[8] C.-H. Huang and L. Dolecek, "Analysis of finite alphabet iterative decoders under processing errors," in Proc IEEE Int. Conf. Acoustics, Speech, Sig. Proc., 2013, pp. 5085-5089.

[9] C. Kameni Ngassa, V. Savin and D. Declercq, "Min-Sum-based decoders running on noisy hardware", in Proc. IEEE GLOBECOM, Atlanta, USA, December 2013.

[10] D. J. C. MacKay, "Good error-correcting codes based on very sparse matrices," IEEE Transactions on Information Theory, vol. 45, no. 2, pp. 399-431, Mar. 1999.

[11] X.-Y. Hu, "Low-delay low-complexity error correcting codes on sparse graphs," Doctoral dissertation, Swiss Federal Institute of Technology Lausanne (EPFL), Lausanne, Switzerland, 2002.

[12] Y. Kou, S. Lin, and M. Fossorier, "Low-density parity-check codes based on finite geometries: a rediscovery and new results," IEEE Transactions on Information Theory, vol. 47, no. 7, pp. 2711-2736, Nov. 2001

[13] J. L. Kim, U. N. Peled, I. Perepelitsa, and V. Pless, "Explicit construction of families of LDPC codes with girth at least six," in Proc. 40th Annu. Allerton Conf. Communication, Control and Computing, Monticello, IL, vol. 40, Part 2, 2002, pp. 1024-1031.

[14] M. Tanner, D. Sridhara, A. Sridharan, T. E. Fuja, and D. J. Costello, Jr., "LDPC block and convolutional codes based on circulant matrices," IEEE Transactions on Information Theory, vol. 50, no. 12, pp. 2966-2984, Dec. 2004.

[15] S. Myung, K. Yang, and J. Kim, "Quasi-Cyclic LDPC Codes for Fast Encoding", IEEE Transactions on Information Theory, vol. 51, no. 8, pp. $2894-2901$, Aug. 2005.

[16] R. Echard, and C.Shih-Chun, "The $\pi$-rotation low-density parity check codes", in Proc IEEE Globecom 2001, vol. 2, 25-29 Nov. 2001, pp. 980-984.

[17] Z. Li, L. Chen, L. Zeng, S.Lin, and W. H. Fong, "Efficient Encoding of Quasi-Cyclic Low-Density Parity-Check Codes", IEEE Transactions on Communications, vol. 54, no. 1, Jan. 2006, pp. 71-81.

[18] M. Lentmaier, D. V. Truhachev, K. S. Zigangirov, and D. J. Costello, Jr., "An analysis of the block error probability performance of iterative decoding," IEEE Transactions on Information Theory, vol. IT-51, no. 11, pp. 3834-3855, Nov. 2005.

[19] A. Shokrollahi, "An Introduction to Low-Density Parity-Check Codes," Theoretical Aspects of Computer Science: Advanced Lectures, Springer-Verlag New York, Inc., New York, NY, 2002.

[20] M. Taylor, "Reliable Information Storage in Memories Designed from Unreliable Components", Bell System Technical Journal, 47, pp 2299-2337, 1968.

[21] M. G. Taylor, "Reliable computation in computing systems designed from unreliable components," Bell Syst. Tech. J., vol. 47, no. 10, pp. 2239-2366, Dec. 1968.

[22] A. Kuznetsov, "Information Storage in a Memory Assembled from Unreliable Components", Problems of Information Transmission, vol 9, pp. 254-264, 1973.

[23] B. Vasic and S. K. Chilappagari, "An information theoretical framework for analysis and design of nanoscale fault-tolerant memories based on low-density parity-check codes," IEEE Trans. Circuits Syst. I, Reg. Papers, vol. 54, no. 11, pp. 2438-2446, Nov. 2007.

[24] M. Ivkovic, S. K. Chilappagari, and B. Vasic, "Construction of memory circuits using unreliable components based on low-density parity-check codes," in Proc. IEEE Global Telecommun. Conf., San Fransisco, CA, USA, Nov.27-Dec.1 2006, pp. 1-5.

[25] S. Brkic, P. Ivanis, G. Djordjevic, B. Vasic, "Taylor-Kuznetsov fault-tolerant memories: a survey and results under correlated gate failures", in Proc IEEE TELSIKS 2013, Nis, Serbia, October $16^{\text {th }}$ $19^{\text {th }}, 2013$, pp. 455-462. (invited paper). 